\title{
1 Substrate Binding and Inhibition of the Anion Exchanger 1 Transporter
}

\section{Authors}

4 Michael J. Capper ${ }^{1}$, Shifan Yang ${ }^{2,3}$, Alexander C. Stone ${ }^{2,3}$, Sezen Vatansever ${ }^{2,3}$,

5 Gregory Zilberg ${ }^{4}$, Yamuna Kalyani Mathiharan ${ }^{1,4}$, Raul Habib ${ }^{1}$, Keino Hutchinson ${ }^{1}$,

6 Avner Schlessinger ${ }^{1}$, Mihaly Mezei ${ }^{1}$, Roman Osman ${ }^{1}$, Bin Zhang ${ }^{1,2,3}$, and Daniel

7 Wacker ${ }^{1,4, *}$

$8 \quad$ Affiliations

9 'Department of Pharmacological Sciences, Icahn School of Medicine at Mount Sinai,

10 New York, New York 10029

$11{ }^{2}$ Department of Genetics and Genomic Sciences, Icahn School of Medicine at Mount

12 Sinai, One Gustave L. Levy Place, New York, NY 10029, USA

$13{ }^{3}$ Mount Sinai Center for Transformative Disease Modeling, Icahn School of Medicine at

14 Mount Sinai, One Gustave L. Levy Place, New York, NY 10029, USA

$15{ }^{4}$ Department of Neuroscience, Icahn School of Medicine at Mount Sinai, New York, New

16 York 10029 
Abstract: Anion Exchanger $1(\mathrm{AE} 1, \mathrm{SLC} 4 \mathrm{~A} 1)$ is the primary bicarbonate $\left(\mathrm{HCO}_{3}^{-}\right)$

22

transporter expressed in erythrocyte membranes where it mediates transport of $\mathrm{CO}_{2}$

between lungs and other tissues via import/export of bicarbonate. It is also a key regulator of erythrocyte structure and antigenic recognition. Previous biochemical studies, and a low-resolution crystal structure of the transmembrane domain have provided initial insight into AE1 structure and function. However, key questions remain regarding substrate binding and transport as well as the mechanism of inhibition. The orientation of the intracellular domain as well as the localization of lipid and sterol binding sites also remain enigmatic. We herein present seven novel high resolution cryo-EM structures of the full length human transporter in the apo, bicarbonate-bound, and several inhibitor-bound states combined with uptake- and computational studies. To our knowledge, these studies represent the first full length human, and substrate bound, SLC4 transporter structure. Our results reveal important molecular details about substrate binding and transport, as well as the diverse mechanisms of AE1 inhibition by both research chemicals and prescription drugs. We also provide novel insights into the full-length transporter architecture, identify the conformational space of the Diego blood antigen system and elucidate multiple lipid and sterol binding sites. 
Main Text:

\section{Introduction}

45 Anion exchanger 1 (AE1); or SLC4A1 is one of nine bicarbonate transporters in the SLC4 family of membrane proteins that help regulate cellular $\mathrm{pH}$ in virtually all tissues. $A E 1$ is a key transporter in red blood cells (RBC) where it shuttles $\mathrm{CO}_{2}$ between lungs and other tissues via bicarbonate transport and contributes to the structural integrity of RBCs through interactions with the cytoskeleton. AE1's extracellular surface binds antibodies of the Diego antigen system, a blood group of 21 antigens that can cause potentially fatal hemolytic disease of the newborn ${ }^{1}$ : Antibodies produced by the mother can bind epitopes on AE1, thereby attacking RBCs of the fetus or the newborn². Other mutations can disrupt $\mathrm{AE} 1$ structure and/or transport resulting in red blood cell deformities and kidney diseases such as renal acidosis ${ }^{3,4}$.

AE1 is modular, but the interplay between its cytoplasmic domain (cdAE1), which binds the cytoskeleton, and membrane domain (mdAE1), which mediates substrate transport, remains poorly understood. AE1 is thought to serve as a nucleus in the periphery of the RBC plasma membrane, forming complexes with enzymes and effectors involved in a variety of $\mathrm{RBC}$ biology ${ }^{5,6}$. $\mathrm{mdAE} 1$ is the functional site of electroneutral $\mathrm{Cl}^{-} / \mathrm{HCO}_{3}-$ exchange through an alternating access mechanism containing a single anion binding site, the precise nature of which is still unknown ${ }^{3}$. A diverse collection of chemical compounds, including clinical drugs, has been shown to inhibit $A E 1$, but the distinct mechanisms by which these compounds inhibit $A E 1$ have been limited to early NMR studies of substrate binding ${ }^{4,7-10}$. 
Although several studies have provided insight into the roles of specific residues in the transport and inhibition of the transporter, key questions regarding inhibitory modes and global molecular mechanisms remain. Answers have been elusive due to the absence of structural insight into $A E 1$, which has been limited to a single lowresolution crystal structure of $\operatorname{mdAE} 1^{11}$. High-resolution cryo-EM has provided the opportunity to elucidate the bicarbonate binding site, as well as to characterize how diverse chemical inhibitors bind to and inhibit AE1. Herein, we report seven unique AE1 cryo-EM structures at resolutions ranging between 2.96-3.37 Å. Our studies allow for

73 the unambiguous near-atomic characterization of the mdAE1 including the Diego

74 antigens, bound lipids, sterols, substrates, and inhibitors, and provide insight into the 75 interplay between the cytoplasmic and transmembrane domains of AE1.

76

77

\section{Results}

\section{Structure Determination of full-length human AE1}

Cryo-EM studies were carried out using the full length human AE1 purified from Sf9 insect cells, and complexed with inhibitors, substrate, or in the "apo" state (see methods for details). Core areas of the structures reach local resolutions as high as $2.5 \AA$ as calculated by local resolution estimation in cryoSPARC (Extended Data Figure 1, Extended Data Table 1). Our highest resolution structure of AE1 bound to the stilbene inhibitor DIDS (4,4'-diisothiocyanatostilbene-2,2'-disulfonic Acid) was obtained at 2.96 $\AA$, and allowed us to identify several key features of the transporter not observed in the previous mdAE1 crystal structure ${ }^{3,11}$. 
Overall, our structures conform with known SLC4 architecture; two AE1

87 protomers form a homodimeric complex (Fig 1). However, unlike previous SLC4 transporter structures ${ }^{11-13}$, our full-length $A E 1$ reconstruction reveals the relative orientation of cdAE1 and mdAE1 (Fig 1, Extended Data Figure 1C). In our resulting model cdAE1 orients perpendicular to mdAE1. The lack of high-resolution information

91 for the cdAE1 implies considerable flexibility between the domains as previously

92 proposed $^{14}$, but indicates that in the absence of binding partners, full-length AE1 protomers neither dimerize in a parallel nor a twisted orientation as has been debated ${ }^{15}$. Extensive 3D sub-classification was unable to produce sufficient density to build an atomic model of cdAE1, which further highlights the flexibility and dynamic nature of cdAE1 in the absence of binding partners. When refined without symmetry, a tilt was present in the cdAE1 that revealed non-covalent contacts between mdAE1 helices 5 and 6 and residues within the cdAE1 (Extended Data Fig 1C). Both, simulations ${ }^{15}$ and cross-linking experiments ${ }^{16}$ have proposed similar interactions, but our structure refutes both protomers forming a tight complex at the same time.

We further reveal the complete extracellular surface of the transporter (Fig 1)

102 including all extracellular loop regions and glycosylation sites of both protomers. We were able to map the position and architecture of all Diego blood group antigens located on AE1 (Extended Data Figure 1D), revealing the detailed molecular architecture of the epitopes targeted in various hemolytic diseases ${ }^{1,2,17}$. density remains unidentified, but we observe three cholesterol molecules per protomer 
109

110

111

112

113

114

115

116

117

118

119

120

121

122

123

124

125

126

127

128

129

130

131

interface of the gate and core domain (TM1 and TM7). Such binding could allosterically modulate the conformational changes required for transport and explain the inhibitory effect of cholesterol on $A E 1^{18-20}$. We also identify two phospholipids bound within the dimer interface between mdAE1 protomers, with the head groups interacting with the extracellular side of AE1 (Fig 1, Extended Data Figure 1).

\section{The Bicarbonate Binding Site}

To better understand the substrate binding mechanism, we determined structures of "apo" and bicarbonate-bound AE1 (Fig 2). "Apo" refers to AE1 purified in the presence of $100 \mathrm{mM}$ chloride, the structure of which does not show any density for chloride near the presumed anion binding site or elsewhere. In contrast, AE1 purified in the presence of $100 \mathrm{mM}$ sodium bicarbonate in a chloride-free buffer (see methods), showed strong electron density attributed to bicarbonate (Fig 2, Extended Data Fig 2). Our near-atomic resolution structure is the first structure of any human SLC4 transporter to reveal the precise binding site of a substrate. In both "apo" and bicarbonate-bound structures, AE1 appears in the outward-facing state, with a channel-like cavity that is accessible from the extracellular site and leads to a positively charged cation selectivity filter/anion binding site near the ends of TM3 and TM10 (Fig 2) ${ }^{3,21}$. Reminiscent of how Uracil is bound in the SLC26 UraA transporter (Extended Data Fig 2C), density for bicarbonate is located near R730, which has previously been implicated in transport ${ }^{3,11,21}$. The negatively charged bicarbonate ion is bound in a small $23 \AA^{3}$ pocket less than $3 \AA$ from the R730's side chain, indicating a strong ionic interaction. We observe weaker interactions between bicarbonate and backbone amide bonds in TM10, which was 
132 relative binding energy contributions of nearby residues in $A M B E R^{22}$ suggests that bicarbonate does not interact with residues at the N-terminal end of TM3, the other proposed dipole of the anion binding site. Moreover, while the R730 sidechain is the key anchor, backbone interactions with T727, T728, and V729 contribute substantially to bicarbonate binding (Extended Data Table 2). Using Simulated Annealing of Chemical

137 Potential (SACP) simulations ${ }^{23}$ (Extended Data Fig 2D), we computationally estimate an apparent bicarbonate $\mathrm{K}_{\mathrm{D}}$ of $1.6 \mathrm{mM}$ for this site, which is similar to NMR studies that estimated a $K_{D}$ of $5.4 \mathrm{mM}^{10}$. To test the relevance of this affinity for AE1-mediated transport, we performed cellular bicarbonate uptake experiments and obtained a concentration of $\mathrm{K}=2.8 \mathrm{mM}$ at which bicarbonate uptake reaches half-saturation (Fig 2G, see methods for details). cavity formed by TM5, TM8, TM10, and TM12 between the core and gate domains of AE1 (Fig 2). This cavity is lined with two serines and constrained by R694 at the

146 cytoplasmic side. These properties and the proximity to bicarbonate suggest that this

147 cavity could expand to become part of a putative substrate exit tunnel in an inward-

148 facing state. In fact, our SACP simulations identify a second bicarbonate binding site in which the anion binds to R694 and S525 (Extended Data Fig 2D), providing further evidence for this proposed exit path.

\section{Molecular mechanisms of transport inhibition}

152 To investigate the structural basis of how different compounds inhibit SLC4-mediated

153 substrate transport, we next determined several structures of AE1 bound to different 154 inhibitors including two clinical drugs. 


\section{Competition for substrate binding}

The stilbene compound $\mathrm{H}_{2}$ DIDS (4,4'-Diisothiocyanatodihydrostilbene-2,2'-

Disulfonic Acid) was used in the previously published mdAE1 structure and appears to be covalently linked to both K539 in TM5 and K851 in TM13. According to previous studies, both lysines are covalently bound at $37^{\circ} \mathrm{C}$ and $\mathrm{pH} 9.5$, while lower $\mathrm{pH}$ prevented linkage of $\mathrm{K} 851^{24}$. Due to some poorly defined electron density in the lower resolution mdAE1- $\mathrm{H}_{2}$ DIDS crystal structure ${ }^{11}$, there indeed remains some ambiguity regarding the bond with $\mathrm{K} 851$. We thus investigated transporter binding by stilbene inhibitors and determined sub $3 \AA$ structures of $A E 1-D I D S$ and $A E 1-H_{2}$ DIDS formed under lesser alkaline conditions $\left(\mathrm{pH} 9,22^{\circ} \mathrm{C}\right)$. Our structures show covalent binding to K539 only, while K851 appears to form ionic interactions with the stilbene's sulfonic acid group and E535 (Fig 3, Extended Data Fig 3). We thus reason that harsher conditions than we used are required to weaken these interactions and facilitate covalent binding to $\mathrm{K} 851$.

When compared with bicarbonate-bound AE1, both DIDS and $\mathrm{H}_{2}$ DIDS are located in the access channel leading from the extracellular space to the buried anion binding site. We further observe that one of the stilbene's sulfonic acid groups is located less than $4 \AA$ from the bicarbonate ion (Fig 3F). These findings indicate that DIDS/ $\mathrm{H}_{2}$ DIDS not only block access to the anion binding site but likely also affect bicarbonate binding through charge repulsion as there remains sufficient space to bind ions. Our findings provide a structural explanation for NMR studies that showed that DIDS reduces substrate affinity. It should be noted that several previous studies 
177 investigated the substrate $\mathrm{Cl}^{-}$not bicarbonate, but competition of both anions for the

178

180

181

182

183

184

185

186

187

same site indicates a common or similar binding site ${ }^{10}$.

We next determined a $3.07 \AA$ structure of $A E 1$ treated with diethyl pyrocarbonate (DEPC) (Extended Data Figure 3), which has been reported to inhibit transport and stilbene binding by stabilizing an inward-facing conformation via covalent modification of H83425,26. Surprisingly, our AE1-DEPC structure shows an outward-facing state, with no electron density accounting for a modified H834 side chain. Instead, we observe that DEPC covalently modifies K539 and K851 (Extended Data Fig 3F-G), indicating that modification of K851 not H834 leads to the increased mass of an AE1 fragment in previous work $^{25}$. We, therefore, suggest that DEPC-mediated modification of K851 sterically precludes $\mathrm{H}_{2}$ DIDS binding rather than stabilizes an inward-facing state ${ }^{26}$.

\section{Inhibition through substrate channel blocking}

While DIDS reduces anion affinity ${ }^{7}$, other inhibitors have been described to only block access to the transport site ${ }^{8}$. One such inhibitor is the FDA-approved antiplatelet medicine dipyridamole, which has been shown to block AE1 substrate channels whilst not competing for anion binding ${ }^{8}$. To elucidate the different mechanisms by which stilbenes and dipyridamole inhibit transport, we determined a 3.19 A structure of AE1dipyridamole (Fig 3, Extended Data Fig 3D). This structure reveals that the drug occupies a similar site as DIDS and $\mathrm{H}_{2}$ DIDS in the same outward-facing transporter conformation. Specifically, dipyridamole stretches between the core and gate domain, where it forms hydrogen bonds with a backbone carbonyl in TM5 and S856 of TM3. One of the piperidine rings appears to stack in the dipole region between TM3 and TM10 towards E681 and closer to TM3. However, dipyridamole binding lacks the 
200

201

202

203

204

205

206

207

208

209

210

211

212

213

214

215

216

217

218

219

220

221

222

charge repulsion provided by the sulfonic acid group of the stilbene compounds, which likely explains why the compound does not compete for anion binding ${ }^{8}$.

\section{Inhibition of translocation}

Niflumic Acid (NIF), an analgesic and anti-inflammatory drug used in the treatment of rheumatoid arthritis, is a chloride channel inhibitor that has previously been shown to inhibit $A E 1$ substrate transport through a mechanism distinct from DIDS, $\mathrm{H}_{2} \mathrm{DIDS}$, or dipyridamole ${ }^{9}$. Specifically, studies have shown that NIF does not affect substrate affinity or access to the binding site, but instead inhibits transport by preventing transition between outward- and inward-facing states ${ }^{9}$. To investigate the molecular basis for this distinct pharmacology, we determined a 3.18 A cryo-EM structure of AE1NIF. Consistent with a different mechanism of action, we observe NIF bound to a different site than $\mathrm{H}_{2}$ DIDS, DIDS, and dipyridamole (Fig 3, Extended Data Figure 4A-E). NIF appears to be accommodated in a $138 \AA^{3}$-sized pocket between the core and gate domains formed by TM3, TM8, TM13, and TM14, which overlaps only partially with dipyridamole's binding pose and the non-attached isothiocyanate groups of DIDS $/ \mathrm{H}_{2}$ DIDS (Fig 3). We also performed molecular docking studies, which further validated NIF's unexpected binding pose and location (Extended Data Fig 4C). NIF appears to be anchored by a salt bridge between its carboxylate group and K851. In addition, the compound is wedged tightly between P467 in TM3 and L859 in TM14 causing structural rearrangements to accommodate the compound. We note subtle outward movements of the solvate exposed tips of TM13 and TM14, as well as changes in e.g., F524, L859, and K851 (Extended Data Fig 4D). Despite inhibiting transport akin to DIDS/ $\mathrm{H}_{2}$ DIDS (Extended Data Fig 4F), NIF however does not seem to obstruct 
223 access to the bicarbonate binding pocket (Fig 3H). Our structure thus suggests that NIF

224 binding between the AE1 gate and core domains prevent translocation-related changes,

225 while not interfering with substrate binding 9 .

\section{Discussion}

227 We herein report seven novel high-resolution cryo-EM structures of the full-length

228 human AE1 transporter bound to substrate and multiple different drugs and

229 therapeutics. We provide key insights into the transporter architecture, fully elucidate

230 the Diego blood group antigens associated with severe hemolytic diseases, and identify

231 lipid and sterol binding sites. Compared to previous work, our studies reveal a fully

232 ordered extracellular surface, including all Diego blood group antigens and glycosylation

233 sites. This observation is surprising as AE1's extracellular surface does not contain

234 secondary structure elements compared to e.g., NDCBE (SLC4A8) ${ }^{13}$. We also reveal

235 several bound lipids and sterols, whose effects on AE1 structure and function have

236 been heavily speculated on ${ }^{19,27,28}$. Contrasting previous studies that propose cholesterol

237 at the dimer interface, we only observe cholesterol bound on membrane-facing

238 surfaces $^{19}$. But we do observe lipids bound to the dimer interface which has been

239 proposed to stabilize and regulate the structure-function of the transporter ${ }^{19}$.

At global resolutions of up to $2.96 \AA$, we also illuminate in near-atomic detail how

241 the substrate bicarbonate binds to AE1, and structurally characterize how chemically

242 and pharmacologically distinct inhibitors differentially affect both substrate binding and

243 transport (Fig 4). Based on structures, uptake assays, and computational studies we

244 propose that R730 forms the center of the anion binding site and holds the anion in

245 place with low millimolar affinity before conformational transition and substrate 
246 translocation. Our observed bicarbonate binding site is similar to that identified in

247 computational modelling ${ }^{29}$, but it is distinct from that of a related sodium-dependent

248 bicarbonate transporter NDCBE (SLC4A8) ${ }^{13}$. However, we have limited structural

249 comparison due to this structure's problematic ion placement with several observed

250 clashes and suboptimal bicarbonate residue environment - likely a result of the lower

251 resolution and ambiguous electron density (Extended Data Fig 2E-F).

Analysis of our structures in the context of other transporter structures provides

253 intriguing insights into AE1's transport mechanisms, which have remained largely

254 elusive. When compared to a previous SLC4 borate transporter structure from $A$.

255 thaliana (AtBor 1$)^{30}$ our findings strongly indicate that AE1 transports bicarbonate via an

257 EL3 and lipids facilitating dimerization ${ }^{19}$ argue for a stationary gate domain that is

258 consistent with an elevator mechanism, but not e.g. a rocker-switch model ${ }^{32}$, and has

259 been described as a common feature of oligomeric elevator transporters ${ }^{33}$.

260 Superposition of AE1 with the AtBor1 structure shows that the gate domains align well,

261 while the AtBor1 core domain appears shifted downward (Fig 4B). TM3 and TM10,

262 which form the AE1 bicarbonate binding site, move downwards by about 5-7 $\AA$, and

263 TM10 bends away from the gate domain, thus likely releasing bound substrate towards

264 the intracellular site. This is further supported by a cytoplasmic exit channel in AtBor1

265 that connects to the AE1 bicarbonate binding site even before translocation of AE1's

266 core domain. A cavity observed in our AE1 structures (Fig 2F) overlaps well with this

267 channel, and likely expands into a substrate exit channel during substrate translocation.

268 In fact, our studies suggest that R694 located at the cytoplasmic exit could form a 
second bicarbonate binding site. Further evidence for this transport model comes from a cholesterol bound between the core and gate domain at the interface of TM1 and TM7 (Fig 1), which may prevent translocation-related conformational changes and explain the inhibitory effects of cholesterol ${ }^{18,20}$. Similarly, although NIF does not appear to inhibit bicarbonate binding, its location between the core and gate domain likely prevents relative domain movements required for ion transport. These findings together with the proposed shared substrate binding site $^{10}$ suggest a general exchange mechanism in which bicarbonate or chloride is transported one way, before the counterion is transported the opposite way and $\mathrm{AE} 1$ is returned to its resting state.

The herein presented work thus not only reveals fundamental mechanisms of AE1-mediated substrate binding and transport, but also illuminates the different pharmacological mechanisms by which distinct research compounds and clinically used drugs such as Niflumic acid and dipyridamole inhibit transport. In fact, dipyridamole increases red blood cell deformability ${ }^{34}$ thus facilitating healthy red blood cell circulation, an effect likely mediated by $A E 1$ 's role in shaping RBC structure ${ }^{35}$. Given AE1's physiological importance in $\mathrm{RBC}$-mediated $\mathrm{CO}_{2}$ transport and acid secretion in the kidney, our structural insights should greatly facilitate the design of novel pharmacological tools to study distal renal tubular acidosis, hemolytic anemias, and other AE1-associated pathologies. Moreover, due to the similar molecular mechanisms of the SLC4, SLC26, and SLC23 anion transporter families, our findings will also inform mechanistic studies of other important transporters in human health and disease. 

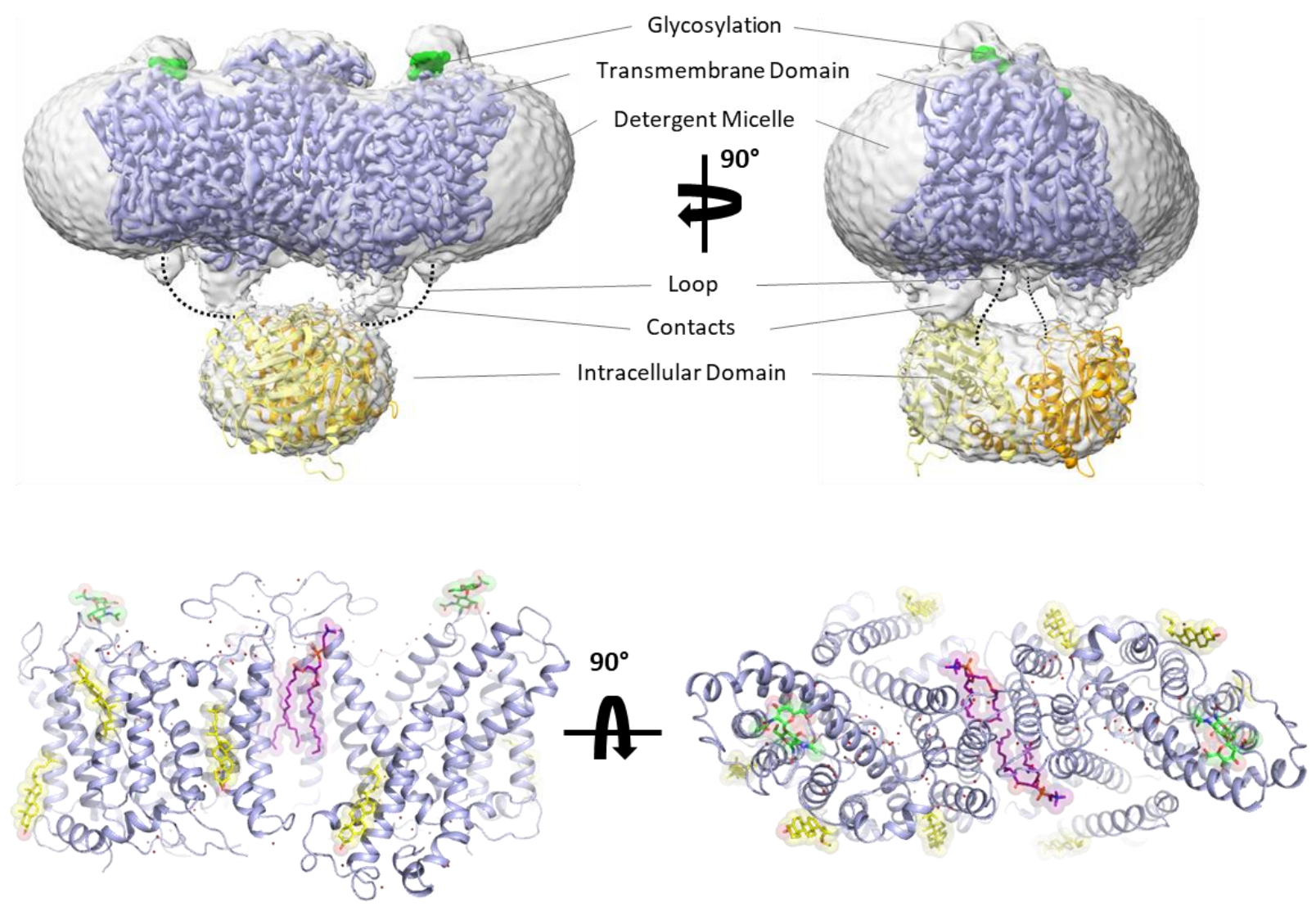

Fig. 1 | Cryo-EM structure of full length human AE1/SLC4A1. Top, Cryo-EM density of overall $A E 1$ homodimer and detergent micelle (grey), overlaid with density of the

294 membrane domain of AE1 (mdAE1, light blue). Glycosylation sites are highlighted in 295 green, and the crystal structure of cytoplasmic domain (cdAE1) homodimer 296 (yellow/orange) is loosely fit into density. Dotted lines highlight that the loop connecting 297 cdAE1 and mdAE1 termini is in a different position from non-covalent contacts observed 298 between the domains. Bottom, mdAE1 structure (light blue) including bound lipids 299 (purple) and cholesterol (yellow), glycosylation sites (green), and water molecules (red 300 spheres). 

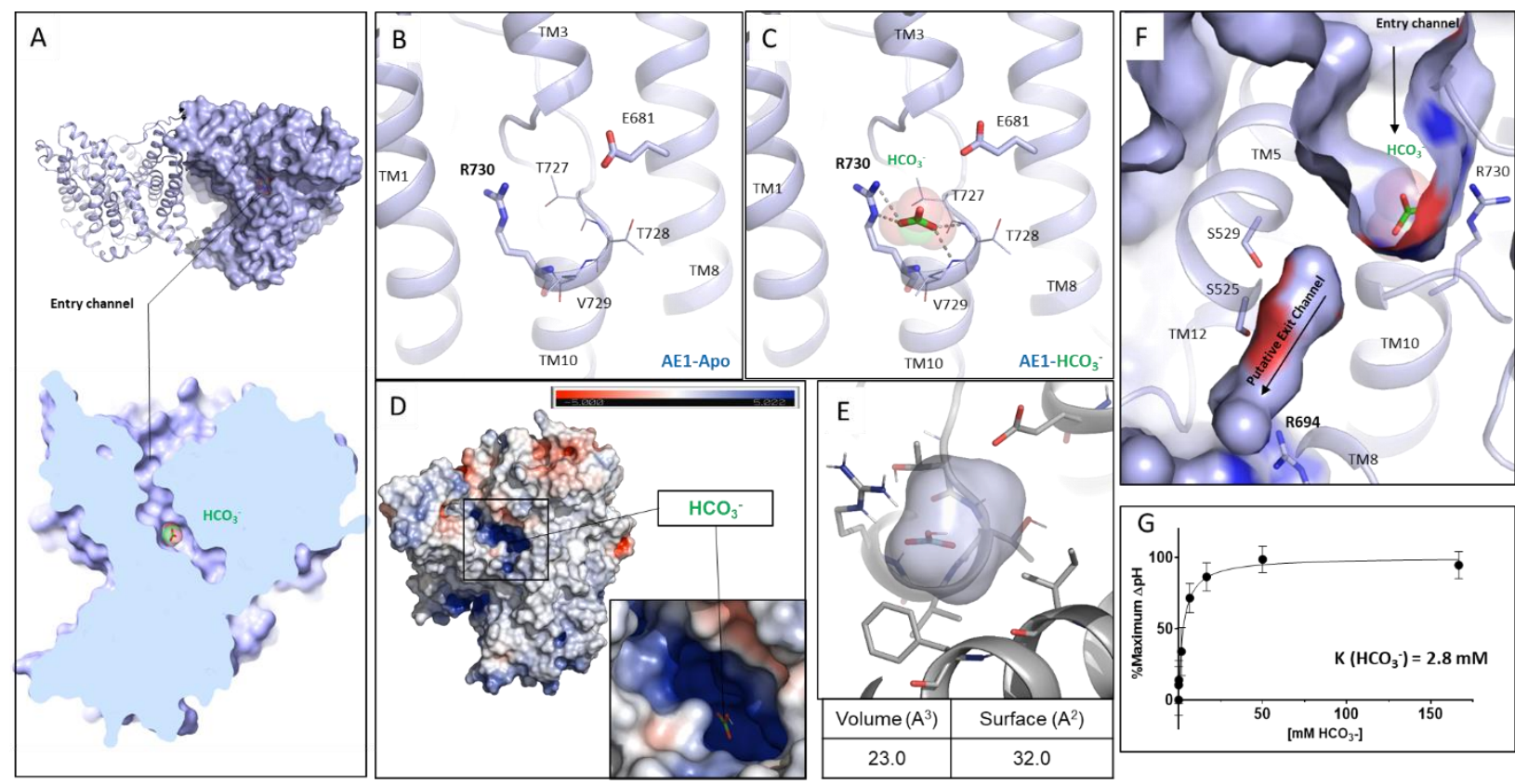

Fig. 2 | Structural insight into bicarbonate binding at AE1. A, Overall structure of mdAE1 homodimer (top), and cut site view of monomer showing bound bicarbonate (bottom, green). B, C, Close-up of anion binding site in apo and bicarbonate bound AE1 structures with highlighted key residues. D, mdAE1 charge distribution highlighting positively (blue) and negatively charged surfaces (red). E, Calculation of bicarbonate binding site volume and surface using POVME. F, Surface display shows putative anion exit channel leading from the bicarbonate binding site to the cytoplasmic site. $\mathbf{G}$, Measurement of cellular bicarbonate uptake over one minute using different substrate concentrations. An uptake constant of $\mathrm{K}=2.8 \mathrm{mM}$ was determined at which concentration half maximum uptake saturation was observed. Data are mean \pm s.e.m. of three independent experiments $(n=3)$ performed in triplicates, and were fit with a onesite saturation curve in GraphPad Prism. 

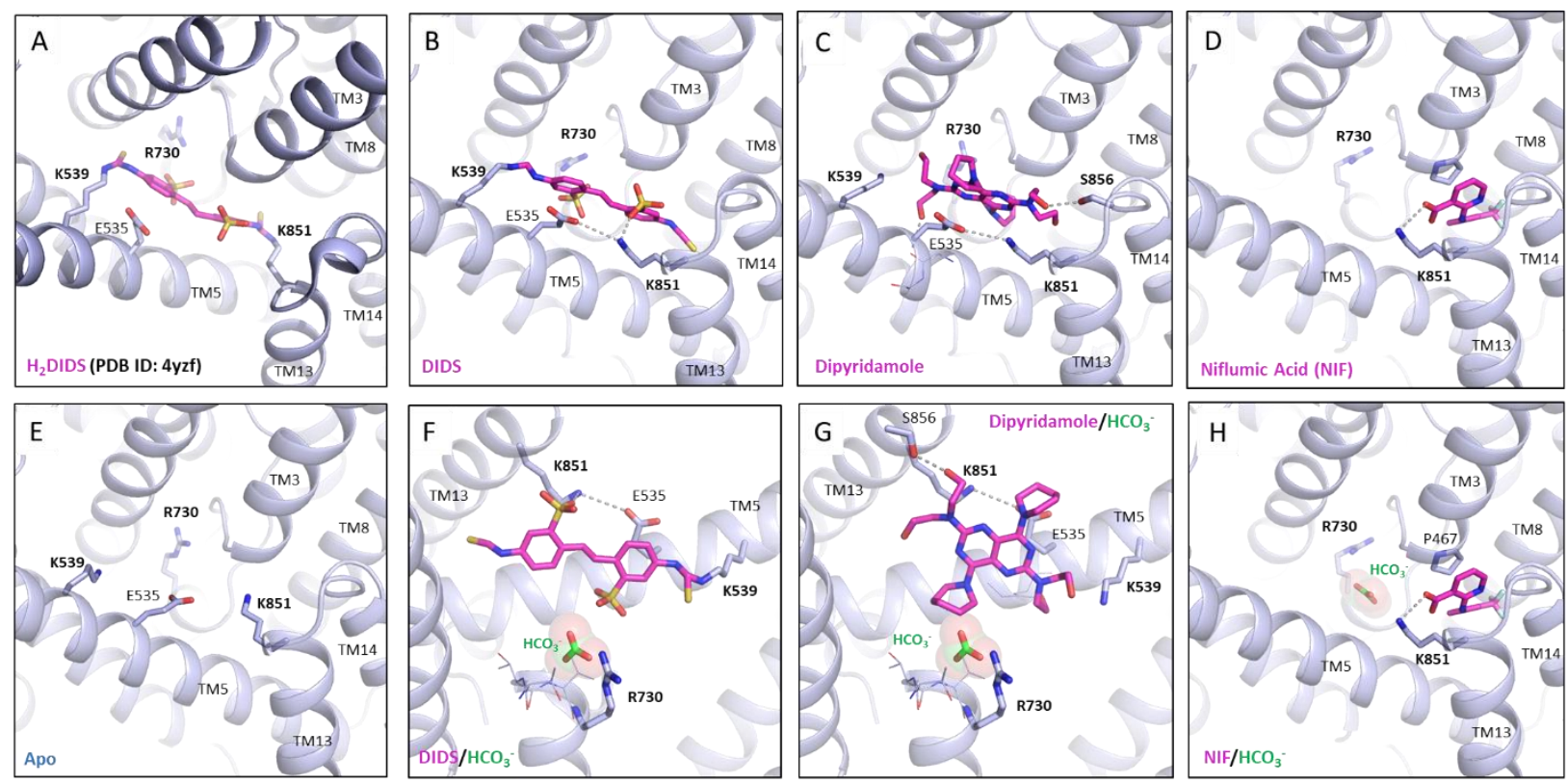

Fig. 3 | Structure of AE1 bound to chemically and pharmacologically diverse

318 inhibitors. A, Previous mdAE1-H2DIDS crystal structure (PDB ID: 4YZF) showing likely covalent binding of $\mathrm{H}_{2}$ DIDS (magenta) to K539 and K851. Cryo-EM structures of AE1 bound to DIDS (B), Dipyridamole (C), NIF (D), or Apo

(E) reveal the binding location and pose of inhibitors (magenta). Overlay with bicarbonate (green) bound AE1 structure shows that DIDS (E) and Dipyridamole (F) NIF (G) binds in a different location and leaves access to anion binding site unobstructed. Ionic interactions and hydrogen bonds are shown as dotted lines. 


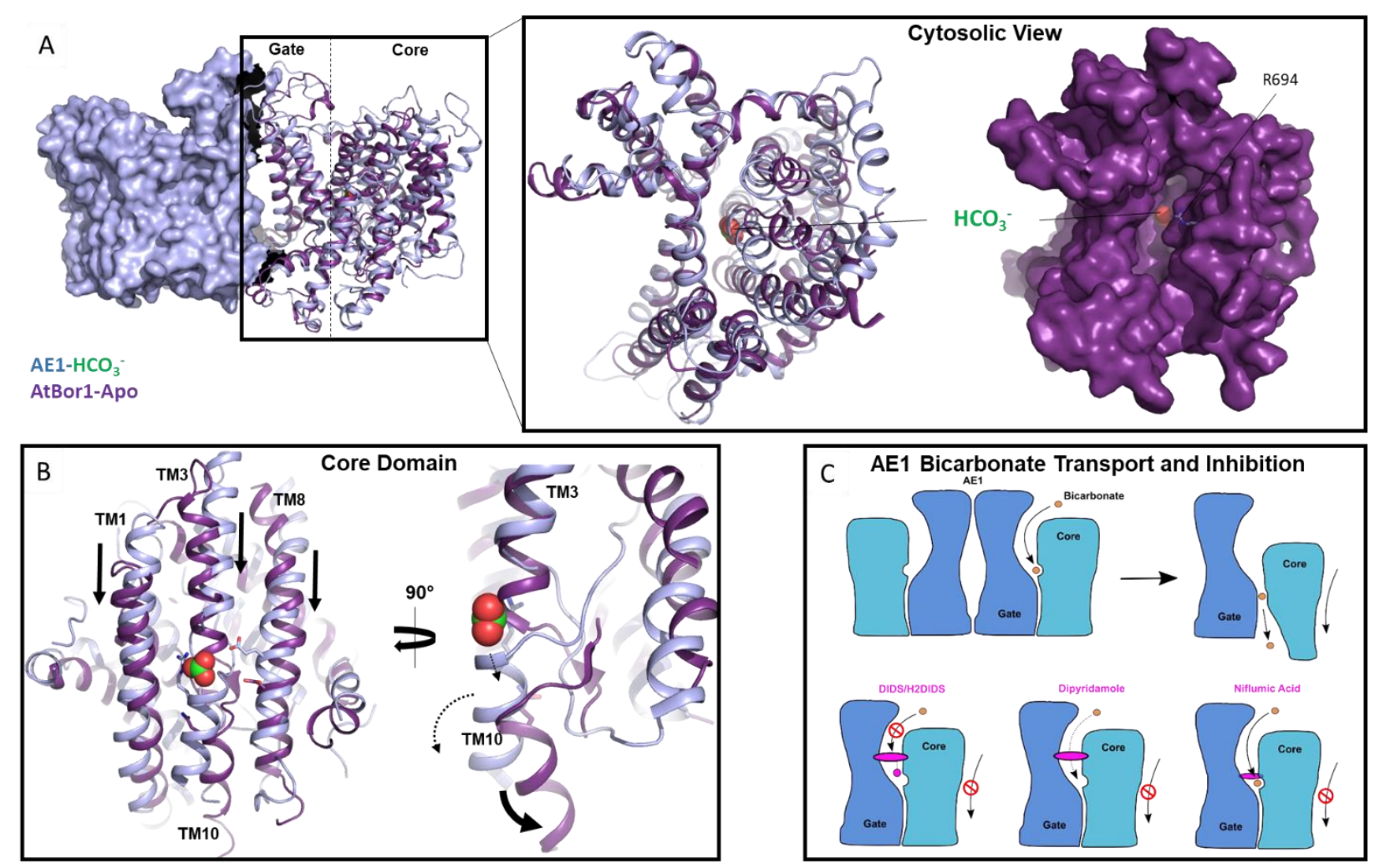

Fig. 4 | Model of AE1-mediated bicarbonate transport and diverse mechanisms of AE1 transport inhibition by pharmacologically different drugs. A, Overlay of membrane domains of human AE1 (light blue) and AtBor1 from A. thaliana (purple) showing similar gate conformation, while the core domains appear in different states. Cytoplasmic view of the overlay reveals a channel in AtBor1 that overlaps with the putative anion exit channel in AE1 ending near R694, and connecting the AE1 bicarbonate binding site and the cytosol. B, Overlay of AE1 and AtBor1 anion binding sites suggests an elevator mechanism where the core domain moves towards the cytoplasmic site (black arrows) and TM10 kinks away from the gate domain (black arrow) to release substrates towards the cytoplasm (dashed arrows). C, Schematic illustrating AE1-mediated bicarbonate transport, as well as pharmacological differences between the inhibitors DIDS $/ \mathrm{H}_{2}$ DIDS, Dipyridamole, and Niflumic Acid. AE1 gate and core domains are shown in blue and teal, respectively, bicarbonate is shown in orange, and inhibitors are colored magenta. 


\section{References}

3471 Poole, J. The Diego blood group system-an update. Immunohematology 15, 135-143 (1999).

3482 Levine, P., Robinson, E. A., Layrisse, M., Arends, T. \& Domingues Sisco, R. The Diego blood factor.

$349 \quad$ Nature 177, 40-41, doi:10.1038/177040b0 (1956).

3503 Reithmeier, R. A. et al. Band 3, the human red cell chloride/bicarbonate anion exchanger (AE1, SLC4A1), in a structural context. Biochim Biophys Acta 1858, 1507-1532,

352 doi:10.1016/j.bbamem.2016.03.030 (2016).

3534 Jennings, M. L. Cell physiology and molecular mechanism of anion transport by erythrocyte band

354

355 3/AE1. Am J Physiol Cell Physiol 321, C1028-C1059, doi:10.1152/ajpcell.00275.2021 (2021).

$5 \quad$ Kodippili, G. C. et al. Analysis of the mobilities of band 3 populations associated with ankyrin protein and junctional complexes in intact murine erythrocytes. J Biol Chem 287, 4129-4138, doi:10.1074/jbc.M111.294439 (2012).

Thornell, I. M. \& Bevensee, M. O. Regulators of SIc4 bicarbonate transporter activity. Front Physiol 6, 166, doi:10.3389/fphys.2015.00166 (2015). Falke, J. J. \& Chan, S. I. Molecular mechanisms of band 3 inhibitors. 1. Transport site inhibitors. Biochemistry 25, 7888-7894, doi:10.1021/bi00372a015 (1986). Biochemistry 25, 7895-7898, doi:10.1021/bi00372a016 (1986). Falke, J. J. \& Chan, S. I. Molecular mechanisms of band 3 inhibitors. 3. Translocation inhibitors. Biochemistry 25, 7899-7906, doi:10.1021/bi00372a017 (1986). (AE1) studied with 14N nuclear magnetic resonance. Biochim Biophys Acta 1079, 146-151, doi:10.1016/0167-4838(91)90119-k (1991). Arakawa, T. et al. Crystal structure of the anion exchanger domain of human erythrocyte band 3 . Science 350, 680-684, doi:10.1126/science.aaa4335 (2015). transporter NBCe1. Nat Commun 9, 900, doi:10.1038/s41467-018-03271-3 (2018). Wang, W. et al. Cryo-EM structure of the sodium-driven chloride/bicarbonate exchanger NDCBE. Nat Commun 12, 5690, doi:10.1038/s41467-021-25998-2 (2021). reveals a flexible linker connecting the cytoplasmic and membrane domains. PLoS One 8, e55408, doi:10.1371/journal.pone.0055408 (2013). 1 Reveal Specific Domain and Lipid Interactions. Biophys J 117, 1364-1379, doi:10.1016/j.bpj.2019.08.029 (2019). Exchanger 1 Structure and Interactions with Ankyrin-1 Determined by Zero Length Crosslinking of Erythrocyte Membranes. Structure 25, 132-145, doi:10.1016/j.str.2016.11.017 (2017). Daniels, G. L. et al. Blood group terminology 2004: from the International Society of Blood Transfusion committee on terminology for red cell surface antigens. Vox Sang 87, 304-316, doi:DOI 10.1111/j.1423-0410.2004.00564.x (2004). doi:10.1016/0014-5793(82)81295-7 (1982). 
410
Kalli, A. C. \& Reithmeier, R. A. F. Interaction of the human erythrocyte Band 3 anion exchanger 1 (AE1, SLC4A1) with lipids and glycophorin A: Molecular organization of the Wright (Wr) blood group antigen. PLoS Comput Biol 14, e1006284, doi:10.1371/journal.pcbi.1006284 (2018).

20 Gregg, V. A. \& Reithmeier, R. A. Effect of cholesterol on phosphate uptake by human red blood cells. FEBS Lett 157, 159-164, doi:10.1016/0014-5793(83)81137-5 (1983).

21 Zhu, Q. \& Casey, J. R. The substrate anion selectivity filter in the human erythrocyte Cl-/HCO3exchange protein, AE1. J Biol Chem 279, 23565-23573, doi:10.1074/jbc.M401380200 (2004).

22 AMBER 16 (University of California, San Francisco, 2016).

23 Guarnieri, F. \& Mezei, M. Simulated annealing of chemical potential: A general procedure for locating bound waters. Application to the study of the differential hydration propensities of the major and minor grooves of DNA. J Am Chem Soc 118, 8493-8494, doi:DOI 10.1021/ja961482a (1996).

24 Okubo, K., Kang, D., Hamasaki, N. \& Jennings, M. L. Red blood cell band 3. Lysine 539 and lysine 851 react with the same H2DIDS (4,4'-diisothiocyanodihydrostilbene-2,2'-disulfonic acid) molecule. J Biol Chem 269, 1918-1926 (1994).

25 Jin, X. R., Abe, Y., Li, C. Y. \& Hamasaki, N. Histidine-834 of human erythrocyte band 3 has an essential role in the conformational changes that occur during the band 3-mediated anion exchange. Biochemistry 42, 12927-12932, doi:10.1021/bi0350809 (2003).

26 Izuhara, K., Okubo, K. \& Hamasaki, N. Conformational change of band 3 protein induced by diethyl pyrocarbonate modification in human erythrocyte ghosts. Biochemistry 28, 4725-4728, doi:10.1021/bi00437a032 (1989).

27 Maneri, L. R. \& Low, P. S. Structural stability of the erythrocyte anion transporter, band 3, in different lipid environments. A differential scanning calorimetric study. J Biol Chem 263, 1617016178 (1988).

28 Maneri, L. R. \& Low, P. S. Fatty acid composition of lipids which copurify with band 3. Biochem Biophys Res Commun 159, 1012-1019, doi:10.1016/0006-291x(89)92209-2 (1989).

29 Zhekova, H. R. et al. Identification of multiple substrate binding sites in SLC4 transporters in the outward-facing conformation: Insights into the transport mechanism. J Biol Chem 296, 100724, doi:10.1016/j.jbc.2021.100724 (2021).

30 Thurtle-Schmidt, B. H. \& Stroud, R. M. Structure of Bor1 supports an elevator transport mechanism for SLC4 anion exchangers. Proc Natl Acad Sci U S A 113, 10542-10546, doi:10.1073/pnas.1612603113 (2016).

31 Ficici, E., Faraldo-Gomez, J. D., Jennings, M. L. \& Forrest, L. R. Asymmetry of inverted-topology repeats in the AE1 anion exchanger suggests an elevator-like mechanism. J Gen Physiol 149, 1149-1164, doi:10.1085/jgp.201711836 (2017).

32 Colas, C., Ung, P. M. \& Schlessinger, A. SLC Transporters: Structure, Function, and Drug Discovery. Medchemcomm 7, 1069-1081, doi:10.1039/C6MD00005C (2016).

33 Garaeva, A. A. \& Slotboom, D. J. Elevator-type mechanisms of membrane transport. Biochem Soc Trans 48, 1227-1241, doi:10.1042/BST20200290 (2020).

34 Saniabadi, A. R. et al. Dipyridamole increases human red blood cell deformability. Eur J Clin Pharmacol 42, 651-654, doi:10.1007/BF00265931 (1992).

35 Gimsa, J. \& Ried, C. Do band 3 protein conformational changes mediate shape changes of human erythrocytes? Mol Membr Biol 12, 247-254, doi:10.3109/09687689509072424 (1995).

36 Zheng, S. Q. et al. MotionCor2: anisotropic correction of beam-induced motion for improved cryo-electron microscopy. Nat Methods 14, 331-332, doi:10.1038/nmeth.4193 (2017).

37 Punjani, A., Rubinstein, J. L., Fleet, D. J. \& Brubaker, M. A. cryoSPARC: algorithms for rapid unsupervised cryo-EM structure determination. Nat Methods 14, 290-296, doi:10.1038/nmeth.4169 (2017). 
Punjani, A., Zhang, H. \& Fleet, D. J. Non-uniform refinement: adaptive regularization improves single-particle cryo-EM reconstruction. Nat Methods 17, 1214-1221, doi:10.1038/s41592-02000990-8 (2020).

39 Yamashita, K., Palmer, C. M., Burnley, T. \& Murshudov, G. N. Cryo-EM single-particle structure refinement and map calculation using Servalcat. Acta Crystallogr D Struct Biol 77, 1282-1291, doi:10.1107/S2059798321009475 (2021).

Liebschner, D. et al. Macromolecular structure determination using X-rays, neutrons and electrons: recent developments in Phenix. Acta Crystallogr D Struct Biol 75, 861-877, doi:10.1107/S2059798319011471 (2019).

41 The PyMOL Molecular Graphics System, Version 2.0 (Schrödinger, LLC). receptor by miraculin. Sci Rep 6, 22807, doi:10.1038/srep22807 (2016).

43 Jo, S., Kim, T., Iyer, V. G. \& Im, W. CHARMM-GUI: a web-based graphical user interface for CHARMM. J Comput Chem 29, 1859-1865, doi:10.1002/jcc.20945 (2008). Mezei, M. Simulaid: a simulation facilitator and analysis program. $J$ Comput Chem 31, 26582668, doi:10.1002/jcc.21551 (2010). Sastry, G. M., Adzhigirey, M., Day, T., Annabhimoju, R. \& Sherman, W. Protein and ligand preparation: parameters, protocols, and influence on virtual screening enrichments. J Comput Aided Mol Des 27, 221-234, doi:10.1007/s10822-013-9644-8 (2013). a series of kinase inhibitors using molecular docking and MM-GBSA scoring. J Med Chem 49, 4805-4808, doi:10.1021/jm060522a (2006).

461

462

47 Adams, D. J. Grand Canonical Ensemble Monte-Carlo for a Lennard-Jones Fluid. Mol Phys 29, 307-311, doi:Doi 10.1080/00268977500100221 (1975).

48 Wagner, J. R. et al. POVME 3.0: Software for Mapping Binding Pocket Flexibility. J Chem Theory Comput 13, 4584-4592, doi:10.1021/acs.jctc.7b00500 (2017). 


\section{Methods}

473

474

475

476

477

478

479

480

481

482

483

484

485

486

487

488

489

490

491

492

493

\section{Construct and Expression}

Structural studies reported herein were performed with the full-length human AE1 transporter (UniprotKB-P02730), which was cloned into a modified pFastBac vector to introduce a C-terminal 3C protease cleavage site followed by a 10xHis tag. Bacmid DNA was generated in DH10Bac cells (Invitrogen) and protein was expressed in Sf9 cells (Expression Systems) using the Bac-to-Bac Baculovirus expression system (Invitrogen). 2.5 $\mu \mathrm{g}$ recombinant bacmid DNA and $3 \mu \mathrm{l}$ FuGENE HD Transfection reagent (Promega) in $100 \mu \mathrm{l}$ Sf900 II media (Invitrogen) were added to 500,000 Sf9 cells plated in $2 \mathrm{ml}$ of SF900 II media in wells of a 12-well plate. After 5 days at $27^{\circ} \mathrm{C}$ the supernatant was harvested as $\mathrm{P} 0$ viral stock, and high-titer recombinant $\mathrm{P} 1$ baculovirus ( $>10^{9}$ viral particles per $\mathrm{ml}$ ) was obtained by adding $200 \mu \mathrm{l} \mathrm{P0}$ to $40 \mathrm{ml}$ of 3 $x 10^{6}$ cells $/ \mathrm{ml}$ and incubating cells for 3 days while shaking at $27^{\circ} \mathrm{C}$. Titers were determined by flow cytometric analysis staining P1 infected cells with gp64-PE antibody (Expression Systems). Expression of AE1 for structural studies was carried out by infection of Sf9 cells at a cell density of $2-3 \times 10^{6}$ cells $/ \mathrm{ml}$ with P1 virus at MOI (multiplicity of infection) of 5 . After 48 hrs of shaking at $27^{\circ} \mathrm{C}$, cells were harvested by centrifugation at $48 \mathrm{~h}$ post-infection and stored at $-80^{\circ} \mathrm{C}$ until use.

\section{Protein Purification and grid preparation}

Typically, we purified protein from $\sim 3 \mathrm{~L}$ of expression culture to prepare grids for cryoEM experiments. Insect cell membranes were disrupted by thawing frozen cell pellets in a hypotonic buffer containing $10 \mathrm{mM}$ HEPES pH 7.5, $10 \mathrm{mM} \mathrm{MgCl}, 20 \mathrm{mM} \mathrm{KCl}$ and 
494 home-made protease inhibitor cocktail (500 $\mu \mathrm{M}$ AEBSF, $1 \mu \mathrm{M}$ E-64, $1 \mu \mathrm{M}$ Leupeptin, 495150 nM Aprotinin) (Gold Biotechnology). Total cellular membranes were harvested by

496 ultracentrifugation, and extensively washed by repeated (2-4 times) homogenization

497 and centrifugation in a high osmotic buffer containing $1 \mathrm{M} \mathrm{NaCl}, 10 \mathrm{mM} \mathrm{HEPES} \mathrm{pH} \mathrm{7.5,}$

$49810 \mathrm{mM} \mathrm{MgCl} 2,20 \mathrm{mM} \mathrm{KCl}$ and home-made protease inhibitor cocktail. Purified

499 membranes were directly flash-frozen in liquid nitrogen and stored at $-80^{\circ} \mathrm{C}$ until further 500 use.

501 Purified membranes were resuspended in buffer containing 10 mM HEPES pH 7.5, 10 $502 \mathrm{mM} \mathrm{MgCl}$, $20 \mathrm{mM} \mathrm{KCl}, 150 \mathrm{mM} \mathrm{NaCl}$, home-made protease inhibitor cocktail, and 25 $503 \mu \mathrm{M}$ DIDS or $\mathrm{H}_{2}$ DIDS, or $100 \mu \mathrm{M}$ Dipyridamole or Niflumic Acid for the different AE1504 inhibitor complexes. Complexation was initiated by agitation for $1 \mathrm{hr}$ at room 505 temperature, a step that was skipped for the AE1 "apo", AE1-bicarbonate and AE1506 DEPC samples. Prior to solubilization, membranes were equilibrated at $4{ }^{\circ} \mathrm{C}$ and 507 incubated for $30 \mathrm{~min}$ in the presence of $2 \mathrm{mg} / \mathrm{ml}$ iodoacetamide (Sigma). Membranes 508 were then solubilized in 10 mM HEPES, pH 7.5, $150 \mathrm{mM} \mathrm{NaCl}, 1 \%$ (w/v) n-dodecyl- $\beta$-D509 maltopyranoside (DDM, Anatrace), 0.2\% (w/v) cholesteryl hemisuccinate (CHS,

510 Anatrace), inhibitor, and home-made protease inhibitor cocktail for $2 \mathrm{~h}$ at $4{ }^{\circ} \mathrm{C}$.

511 Unsolubilized material was removed by centrifugation at 200,000 $\times \mathrm{g}$ for $30 \mathrm{~min}$, and

512 buffered imidazole was added to the supernatant for a final concentration of $20 \mathrm{mM}$.

513 Proteins were bound to TALON IMAC resin (Clontech) overnight at $4{ }^{\circ} \mathrm{C}$. Purification of 514 the Dipyridamole and NIF bound complex was carried out in the presence of $50 \mu \mathrm{M}$ 515 inhibitor and the bicarbonate bound complex was purified in the presence of $100 \mathrm{mM}$ 516 sodium bicarbonate. The resin was then washed with 10 column volumes (cv) of Wash 
517 Buffer I (25 mM HEPES, pH 7.5, 500 mM NaCl, 0.1\% (w/v) DDM, 0.02\% (w/v) CHS, 20

$518 \mathrm{mM}$ imidazole, $10 \%(\mathrm{v} / \mathrm{v})$ glycerol). The detergent was then exchanged for LMNG by

519 successively incubating the resin with the following buffers for 1 hour each: Wash Buffer

520 II (25 mM HEPES, pH 7.5, 500 mM NaCl, 0.05\% (w/v) DDM, 0.05\% (w/v) LMNG, 0.02\%

521 (w/v) CHS), Wash Buffer III (25 mM HEPES, pH 7.5, 500 mM NaCl, 0.025\% (w/v) DDM,

$522 \quad$ 0.075\% (w/v) LMNG, 0.02\% (w/v) CHS), Wash Buffer IV (25 mM HEPES, pH 7.5, 500

$523 \mathrm{mM} \mathrm{NaCl}, 0.05 \%$ (w/v) LMNG, 0.02\% (w/v) CHS), Wash Buffer V (25 mM HEPES, pH

$5247.5,500 \mathrm{mM} \mathrm{NaCl}, 0.025 \%$ (w/v) LMNG, 0.02\% (w/v) CHS). After the final incubation

525 step, the proteins were eluted with 25 mM HEPES, pH 7.5, $500 \mathrm{mM} \mathrm{NaCl}, 0.025 \%$ (w/v)

526 LMNG, 0.02\% (w/v) CHS and $250 \mathrm{mM}$ imidazole. Protein purity and monodispersity

527 were tested by SDS-PAGE and analytical size-exclusion chromatography (aSEC).

528 Typically, the protein purity exceeded 95\%, and the aSEC profile showed a single peak,

529 indicative of transporter monodispersity. For the AE1-DEPC sample, we then added 5

$530 \mathrm{mM}$ DEPC and incubated the sample overnight at $4^{\circ} \mathrm{C}$. All complexes were finally

531 purified over a S200 size exclusion chromatography column equilibrated in $20 \mathrm{mM}$

532 HEPES, pH 7.5, 150 mM NaCl, 0.0011\% (w/v) LMNG, 0.00011\% (w/v) CHS, 0.00025\%

533 GDN. For AE1 bound to NIF and Dipyridamole, $50 \mu \mathrm{M}$ of the respective compound was

534 added to the buffer. The bicarbonate complex was purified in $20 \mathrm{mM} \mathrm{HEPES,} \mathrm{pH} 7.5$,

$535100 \mathrm{mM} \mathrm{NaCHO}_{3}$ 0.001\% (w/v) LMNG, 0.0001\% (w/v) CHS, 0.0001\% GDN. Peak

536 fractions were then pooled, concentrated to $\sim 3-7 \mathrm{mg} / \mathrm{ml}$, and immediately used to

537 prepare grids for cryo-EM data collection. 
541 To prepare cryo-EM grids for imaging, $3 \mu \mathrm{l}$ of purified AE1-Apo at $\sim 6.3 \mathrm{mg} / \mathrm{ml}, \mathrm{AE} 1$ -

542 bicarbonate at $5 \mathrm{mg} / \mathrm{ml}, \mathrm{AE} 1$-DIDS at $\sim 5 \mathrm{mg} / \mathrm{ml}, \mathrm{AE} 1-\mathrm{H}_{2}$ DIDS at $\sim 4.1 \mathrm{mg} / \mathrm{ml}, \mathrm{AE} 1$ -

543 DEPC at $5 \mathrm{mg} / \mathrm{ml}, \mathrm{AE} 1$-Dipyridamole at $4.8 \mathrm{mg} / \mathrm{ml}$, or AE1-Niflumic acid at $5 \mathrm{mg} / \mathrm{ml}$

544 were applied to glow-discharged holey carbon EM grids (Quantifoil 300 copper mesh,

$545 \mathrm{R} 1.2 / 1.3$ ) in an EM-GP2 plunge freezer (Leica). EM-GP2 chamber was set to $95 \%$

546 humidity at $12^{\circ} \mathrm{C}$. Sample-coated grids were blotted for 3 to 3.3 seconds before plunge-

547 freezing into liquid ethane and stored in liquid nitrogen for data collection.

All automatic data collection was performed on a FEI Titan Krios equipped with a Gatan

K3 direct electron detector run and operated by the Simons Electron Microscopy Center

551 BioMolecular Structure at Brookhaven National Laboratory. The microscope was

552 operated at $300 \mathrm{kV}$ accelerating voltage, at a nominal magnification of $64,000-81,000$

553 corresponding to a pixel size of $1.08 \AA$. For each dataset, at least 3,500 movies were obtained at a dose rate of 25-30 electrons per $\AA^{2}$ per second with a defocus ranging 555 from -0.5 to $-1.8 \mu \mathrm{m}$. The total exposure time was $2 \mathrm{~s}$ and intermediate frames were recorded in $0.05 \mathrm{~s}$ intervals, resulting in an accumulated dose of 50-60 electrons per $\AA^{2}$ 557 and a total of 40 frames per micrograph.

558 Movies were motion-corrected using MotionCor $2^{36}$ and imported to cryoSPARC for 559 further processing ${ }^{37}$. For CTF estimation we used patchCTF in cryoSPARC. An initial 560 model was produced using a subset of micrographs and manual picking. Subsequent 561 models were produced from particles found using templates. Datasets were curated by 562 the removal of micrographs deemed irredeemable by poor CTF estimation. Particles 
563 were subject to $2 \mathrm{D}$ classification which quickly identified both the moAE1 and cdAE1. A

564 good initial model of mdAE1 was generated using ab-initio model building in

565 cryoSPARC as were several bad models from rejected particles as a sink in future

566 hetero-refinement. Multiple rounds of hetero-refinement were carried out to improve

567 resolution followed by NU-refinement ${ }^{37,38}$. Stuctures were then further refined in

568 ServalCat ${ }^{39}$, and final maps were generated in PHENIX ${ }^{40}$ before import into PyMOL ${ }^{41}$

569 for generating figures shown in the manuscript.

570 Bicarbonate Transport Assay

571 A polyclonal HEK cell line that stably expresses AE1 upon tetracycline induction was

572 generated using the TRex system (Invitrogen). Cells were plated in a 96-well plate and

573 incubated overnight with or without $2 \mu \mathrm{g} / \mathrm{ml}$ tetracycline at $37^{\circ} \mathrm{C}$. The next day, induced

574 cells were again incubated with tetracycline for 3-4 hours. Cellular bicarbonate uptake

575 was then determined via cellular changes in $\mathrm{pH}$ as previously described for other SLC4

576 transporters ${ }^{12}$. Cells were loaded with $5 \mu \mathrm{M}$ of the $\mathrm{pH}$-sensitive fluorescent dye BCECF-

577 AM (2',7'-Bis-(2-Carboxyethyl)-5-(and-6)-Carboxyfluorescein, Acetoxymethyl Ester) for

57830 minutes. Following another short incubation in Hank's balanced salt solution (HBSS)

579 buffered with $50 \mathrm{mM}$ HEPES pH 7.5, intracellular fluorescence ratio (excitation $495 \pm 20$

$580 \mathrm{~nm}$ and $435 \pm 20 \mathrm{~nm}$; emission $540 \pm 30 \mathrm{~nm}$ ) was measured using a multimode plate

581 reader (Victor NIVO, Perkin Elmer). To initiate uptake, cells were then diluted 1:3 in Cl-

582 free buffer (50 mM HEPES pH 7.5 adjusted with $\mathrm{NaOH}, 115 \mathrm{mM}$ Na gluconate, $2.5 \mathrm{mM}$

$583 \mathrm{~K}_{2} \mathrm{HPO}_{4}, 7 \mathrm{mM}$ Ca gluconate, $1 \mathrm{mM}$ Mg gluconate, $5 \mathrm{mM}$ glucose, $30 \mu \mathrm{M}$ amiloride),

584 supplemented with different $\mathrm{NaHCO}_{3}$ concentrations. Fluorescence was then measured

585 after 1 min. A calibration experiment using $10 \mu \mathrm{M}$ nigericin in modified HBSS (1.26 mM 
$\mathrm{CaCl}_{2}, 0.493 \mathrm{mM} \mathrm{MgCl}_{2}, 0.407 \mathrm{mM} \mathrm{MgSO}_{4}, 140 \mathrm{mM} \mathrm{KCl}, 0.441 \mathrm{mM} \mathrm{KH}_{2} \mathrm{PO}_{4}, 4.17 \mathrm{mM}$ $\mathrm{NaHCO}_{3}, 0.338 \mathrm{mM} \mathrm{Na}_{2} \mathrm{HPO}_{4}, 10 \mathrm{mM} \mathrm{HEPES}$ ) at a range of $\mathrm{pH}$ values between 7 and 8 was then performed to convert fluorescence to $\mathrm{pH}$ values ${ }^{42}$. human transporter with up to $10^{5}$ ions per second ${ }^{3}$ ) and cellular changes associated with changing $\mathrm{pH}$, we opted not to determine a $\mathrm{K}_{\mathrm{M}}$. Instead, we set out to determine an uptake constant via a saturatable uptake assay, which was performed in induced and uninduced cells using 0-167 $\mathrm{mM} \mathrm{NaHCO}_{3}$. Specific uptake was then determined by plotting $\mathrm{pH}$ differences between induced and uninduced cells in response to different $\mathrm{NaHCO}_{3}$ concentrations. This was done in GraphPad Prism using a one-site saturation curve and allowed calculation of an uptake constant $\mathrm{K}=2.8 \mathrm{mM}$ at which $\mathrm{NaHCO}_{3}$ concentration $\mathrm{pH}$ differences between induced and uninduced cells were at half maximum.

To measure AE1 inhibition, this experiment was performed in induced and uninduced cells using $16.7 \mathrm{mM} \mathrm{NaHCO}_{3}$. For DIDS and $\mathrm{H}_{2}$ DIDS, cells were preincubated with 20 $\mu \mathrm{M}$ inhibitor in HEPES-buffered HBSS for $1 \mathrm{hr}$, after which DIDS and $\mathrm{H}_{2}$ DIDS were omitted from the experiment. For NIF, $50 \mu \mathrm{M}$ were added throughout the experiment after dye loading. Dipyridamole has spectral overlap with BCECF-AM and could

604 therefore not be included in our measurements. All experiments were performed in 605 triplicates, and data was averaged from three independent experiments and is shown as 
608

609

610

611

612

613

614

615

616

617

618

619

620

621

622

623

624

625

626

627

628

629

630

\section{$\underline{\text { MD simulations }}$}

Even though AE1 exist in a dimeric form, it appears that the functional properties of each monomer are independent of the other. Thus only one monomer was selected for the construction. The system was built with CHARMM-GUI ${ }^{43}$ adding two cholesterol molecules and one cholesteryl succinate in the positions identified in the cryo-EM structure. The membrane was constructed from 200 POPC in both layers divided to account for the different surface area of the protein in the upper and lower leaves. The concentration of neutralizing $\mathrm{K}^{+}$and $\mathrm{Cl}^{-}$counterions in the rectangular box was set to 0.15 mM. The initial apo-AE1 structure was translated using the charmm2lipid routine in $\mathrm{AMBER}^{22}$, and the simulations were conducted in AMBER 20. The system was minimized and equilibrated with the restraints designed in CHARMM-GUI. At the end of the equilibration, the MD simulations were executed at NPT conditions for $1000 \mathrm{~ns}$. The final trajectory included 10,000 structures. A similar design and simulations were performed on the bicarbonate-occupied structure. The analysis of the trajectories were performed with cpptraj in AMBER and the simulaid facility ${ }^{44}$. The RMSD of the protein stabilizes after 100 ns and remains nearly constant for the rest of the simulation.

\section{Molecular Docking}

To characterize the binding mode of NIF at AE1 using molecular docking calculations, we first removed the ligand from the cryo-EM structure of the AE1-NIF complex. AE1 was then prepared with the Maestro Protein Preparation Wizard under default parameters ${ }^{45}$. The binding site was defined by generating a grid with the Receptor Grid Generation Panel. The binding site outlining box was defined around the reference NIF ligand in the AE1 template structure. The NIF compound structure was obtained from 
631 PubChem (PubChem CID: 4488), and it was prepared for docking using LigPrep with

632 the default parameters, where the possible states were generated at target $\mathrm{pH} 7 \pm 2$.

633 Docking was performed using Glide from the Schrödinger suite (Schrödinger, 2021).

634 Finally, we used molecular mechanics generalized with born surface area solvation

635 (MM-GBSA) with Prime in the Schrödinger suite to estimate the relative binding affinity

636 between NIF and $A E 1^{46}$, where a more negative value of $\Delta G$ binding indicates higher

637 binding affinity.

638 Calculation of Bicarbonate Binding Energies

639 To compute the binding energy of bicarbonate we took the approach of Simulated

640 Annealing of Chemical Potential (SACP) ${ }^{23}$. Briefly, the system is placed in a periodic

641 box, which is divided into an inner box whose dimensions are $10 \AA$ beyond the

642 boundaries of the molecule and an outer ("bulk") box of additional $5 \AA$ thickness. Using a

643 Grand Canonical Ensemble/Monte Carlo (GC/MC) approach the entire system is

644 equilibrated with inserting/deleting bicarbonate to reach a density of $0.15 \mathrm{~g} / \mathrm{L}$. The B parameter ${ }^{47}$, which reflects the excess chemical potential $\left(B=\mu_{\varepsilon x}+\ln <N>\right)$ in the "bulk"

646 box is then decreased progressively. The change in the B parameter increases the

647 probability of deletion of bicarbonate until the system equilibrates. The value of the B

648 parameter at the point where the last bicarbonate is deleted equals the most negative

649 energy of bicarbonate to the protein. An analysis of the B value at which bicarbonate is

650 most proximal to a specific site (e.g., R730) yields the affinity of bicarbonate to this site.

651 To enhance the statistical significance of the computed values, the MD trajectory was

652 divided into 10 clusters and the center of the cluster was extracted to perform the SACP 
653 on each of them. The final result is the population weighted average of all the clusters

654 for a specific location of the bicarbonate.

655 Pocket Volume Analysis

656

657

658

659

660

661

662

663

664

665

666

667

668

669

670

671

672

673

674

POVME3 $^{48}$ (Pocket Volume Measurer 3) was used to calculate binding site volumes.

We used default parameters for ligand-defined inclusion region, using the recently resolved structures as input PDBs. Pocket volume was visualized using $\mathrm{PyMOL}^{41}$.

\section{Acknowledgements}

This work was supported by NIH grant GM133504, a Sloan Research Fellowship in Neuroscience, an Edward Mallinckrodt, Jr. Foundation Grant, a McKnight Foundation Scholars Award (all to D.W.), NIH T32 Training Grant GM062754 (G.Z.), R01

GM108911 (A.Sch. and K.H.), NIH Grant U01AG046170 (B.Z.), NIH Grant RF1AG057440 (B.Z.), NIH Grant R01AG068030 (B.Z.). NIH Grants R01DK073681, R01DK067555, R01DK061659 (R.O). Some of this work was performed at the National Center for cryo-EM Access and Training (NCCAT) and the Simons Electron Microscopy Center located at the New York Structural Biology Center, supported by the NIH Common Fund Transformative High Resolution Cryo-Electron Microscopy program (U24 GM129539,) and by grants from the Simons Foundation (SF349247) and NY State Assembly. We further acknowledge cryo-EM resources at the National Resource for Automated Molecular Microscopy located at the New York Structural Biology Center, supported by grants from the Simons Foundation (SF349247), NYSTAR, and the NIH 
675 National Institute of General Medical Sciences (GM103310) with additional support from

676

677

678

679

680

681

682

683

684

685

686

687

688

689

690

691

692

693

694

695

696

Agouron Institute (F00316) and NIH (OD019994). For additional data collection we are also grateful to staff at the Laboratory for BioMolecular Structure (LBMS), which is supported by the DOE Office of Biological and Environmental Research (KP160711). This work was supported in part through the computational resources and staff expertise provided by Scientific Computing at the Icahn School of Medicine at Mount Sinai. We also like to thank J. F. Fay for help with initial data processing.

\section{Author Contributions}

M.J.C. designed experiments, expressed and purified protein for grid freezing, collected data, refined structures, and helped write the manuscript. S.Y. and A.S. purified protein, prepared samples for grid freezing, and performed functional assays. S.V. performed computational studies and helped analyze the structures. G.Z. prepared grids for structure determination and assisted with data collection. Y.K.M. helped with data processing and structure refinement. R.H. helped establish protein expression and purification. S.V, K.H. performed docking studies and volume calculations supervised by A.Sch. R.O. performed molecular simulations and SACP analysis of substrate binding with help from M.M. B.Z. contributed to the study design and supervised computational studies. D.W. designed experiments, analysed the data, supervised the overall project and management, and wrote the manuscript. 
697 Data Availability: Density maps and structure coordinates have been deposited in the

698 Electron Microscopy Data Bank (EMDB) and the PDB: AE1-Apo (EMD-XXXXX and

699 XXX), AE1-Bicarbonate (EMD-XXXXX and XXX), AE1-DIDS (EMD-XXXXX and XXX),

700 AE1-H2DIDS (EMD-XXXXX and XXX), AE1-DEPC (EMD-XXXXX and XXX), AE1-

701 Dipyridamole (EMD-XXXXX and XXX), AE1-NIF (EMD-XXXXX and XXX).

\section{Author Information}

703 Correspondence and requests for materials should be addressed to

704 daniel.wacker@mssm.edu.

705 Competing interests: The authors declare no competing interests

706

707

708

709 


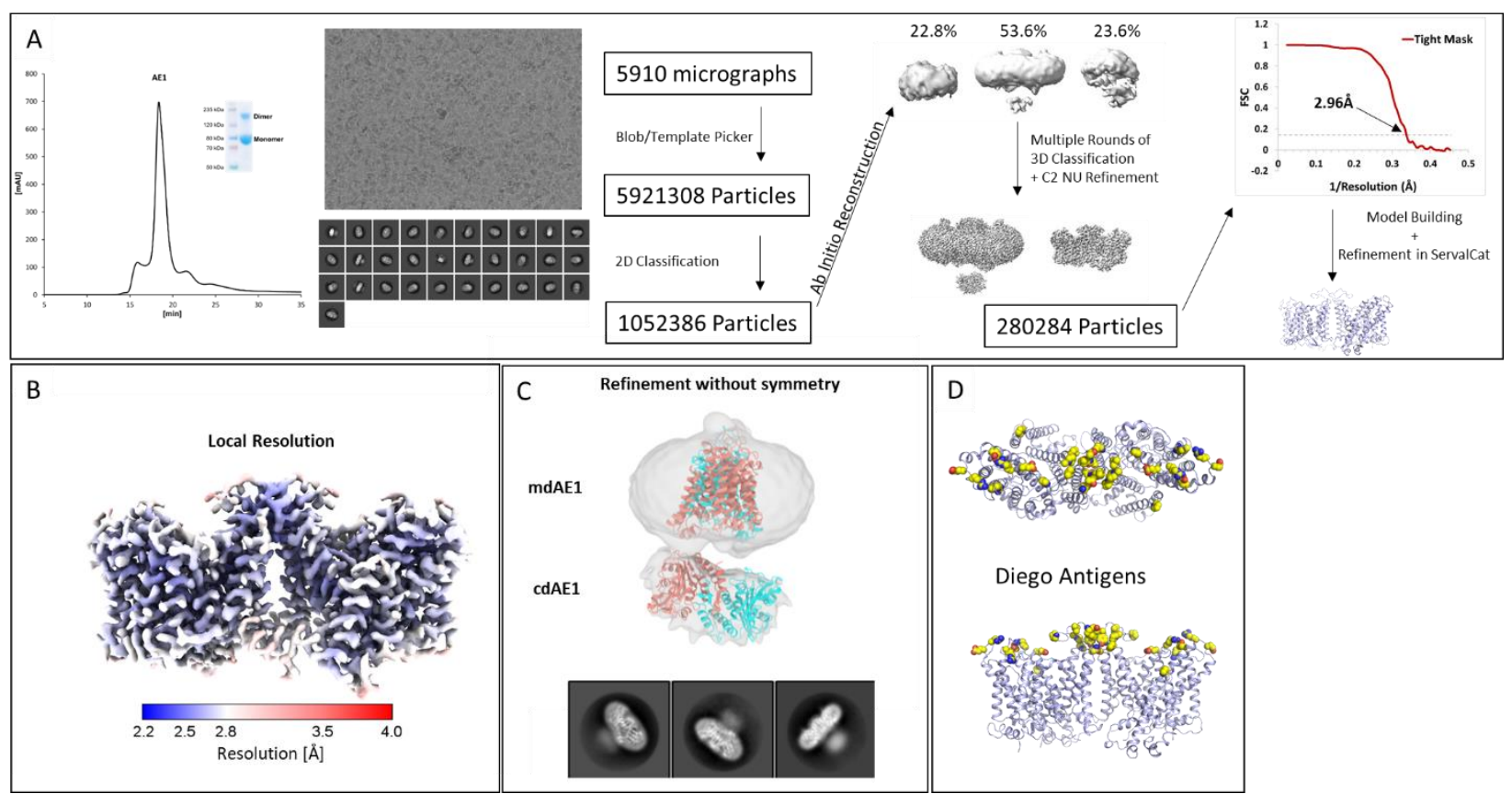

Extended Data Fig. 1 | Workflow of cryo-EM Structure Determination of AE1-DIDS.

A, Analytical size exclusion chromatography and SDS-PAGE show monodisperse and pure protein. Data were collected and processed in cryoSPARC: particles were picked from micrographs, subjected to 2D classification, followed by ab initio 3D classification. After multiple rounds of 3D classification, the final particle stack was refined using nonuniform refinement with imposed C2 symmetry. Final map was obtained with GS-FSC indicating a resolution of $2.96 \AA$ applying the 0.143 cutoff. Initial model was built in PHENIX, and then further refined in ServalCat for the generation of final maps and coordinates of mdAE1. B, Calculations in cryoSPARC indicate local resolutions of up to $2.5 \AA$ around substrate and inhibitor binding sites. C, Example map produced through refinement without symmetry shows tilt between $\operatorname{cdAE} 1$ and $\mathrm{mdAE} 1$, highlighting the

721 flexibility of cdAE1. 2D classes highlight well-ordered mdAE1 density, and poorly 722 defined cdAE1 density, respectively. D, Cryo-EM structures allowed us to build the 723 complete extracellular surface including all Diego antigens (yellow). 

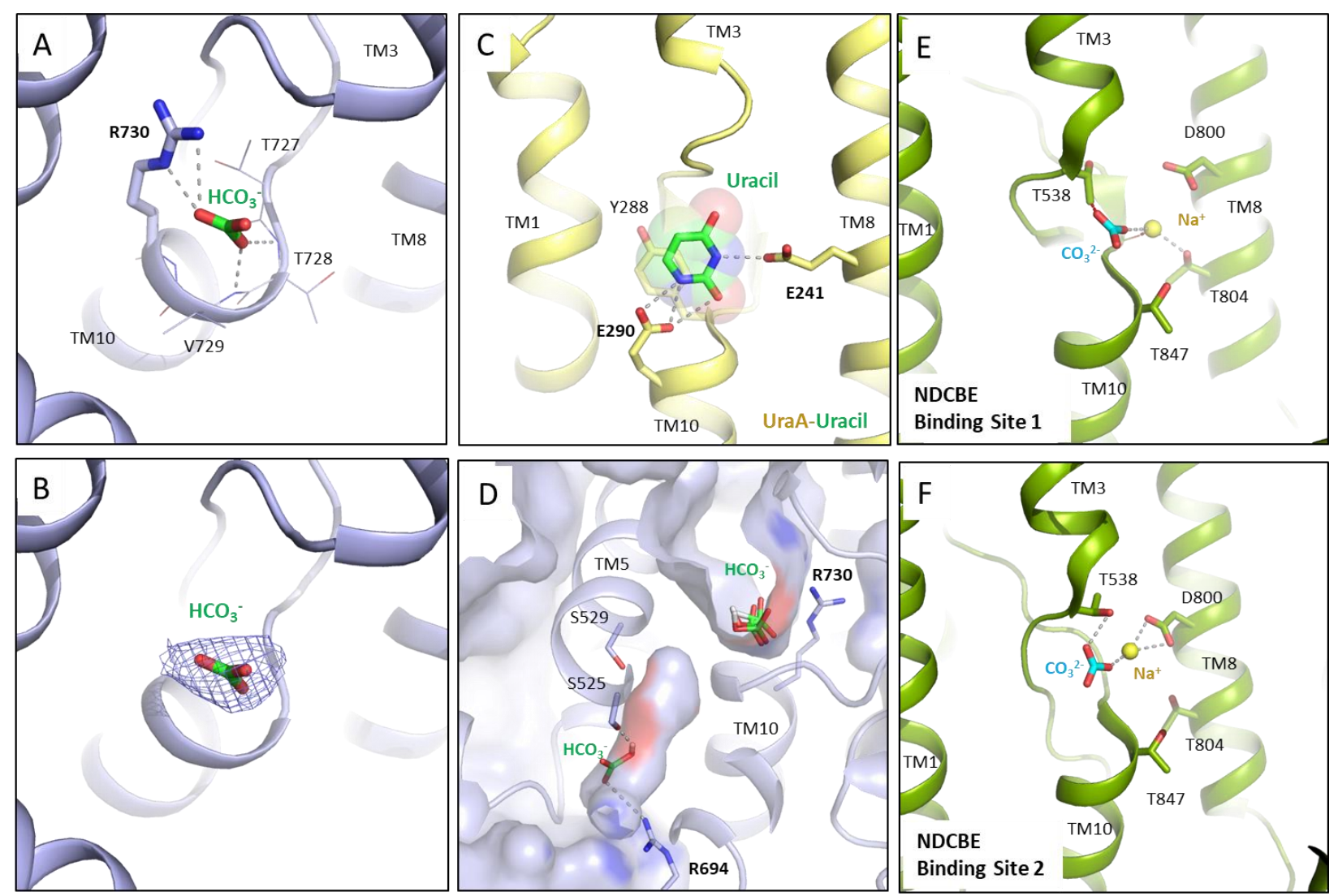

Extended Data Fig. 2 | Substrate-binding in SLC4 and SLC26 transporters. A,B,

Binding site and cryo-EM density of bicarbonate in AE1-bicarbonate structure using a contour level of $6 \sigma$. C, Binding site of uracil in the UraA-Uracil structure (PDB ID: 3QE7). D, MD simulations allow to calculate bicarbonate affinity to AE1 anion binding site and identify a second bicarbonate binding site in the putative exit channel. E,F, Carbonate and sodium ions in the rabbit NDCBE (SLC4A8) cryo-EM structure (PDB ID: 7RTM, EMD-24683), with clashes between $\mathrm{CO}_{3}{ }^{2-}$ and T538, as well as $\mathrm{Na}^{+}$and the backbone shown as red dotted lines. Ionic interactions and hydrogen bonds are shown as grey dotted lines. 

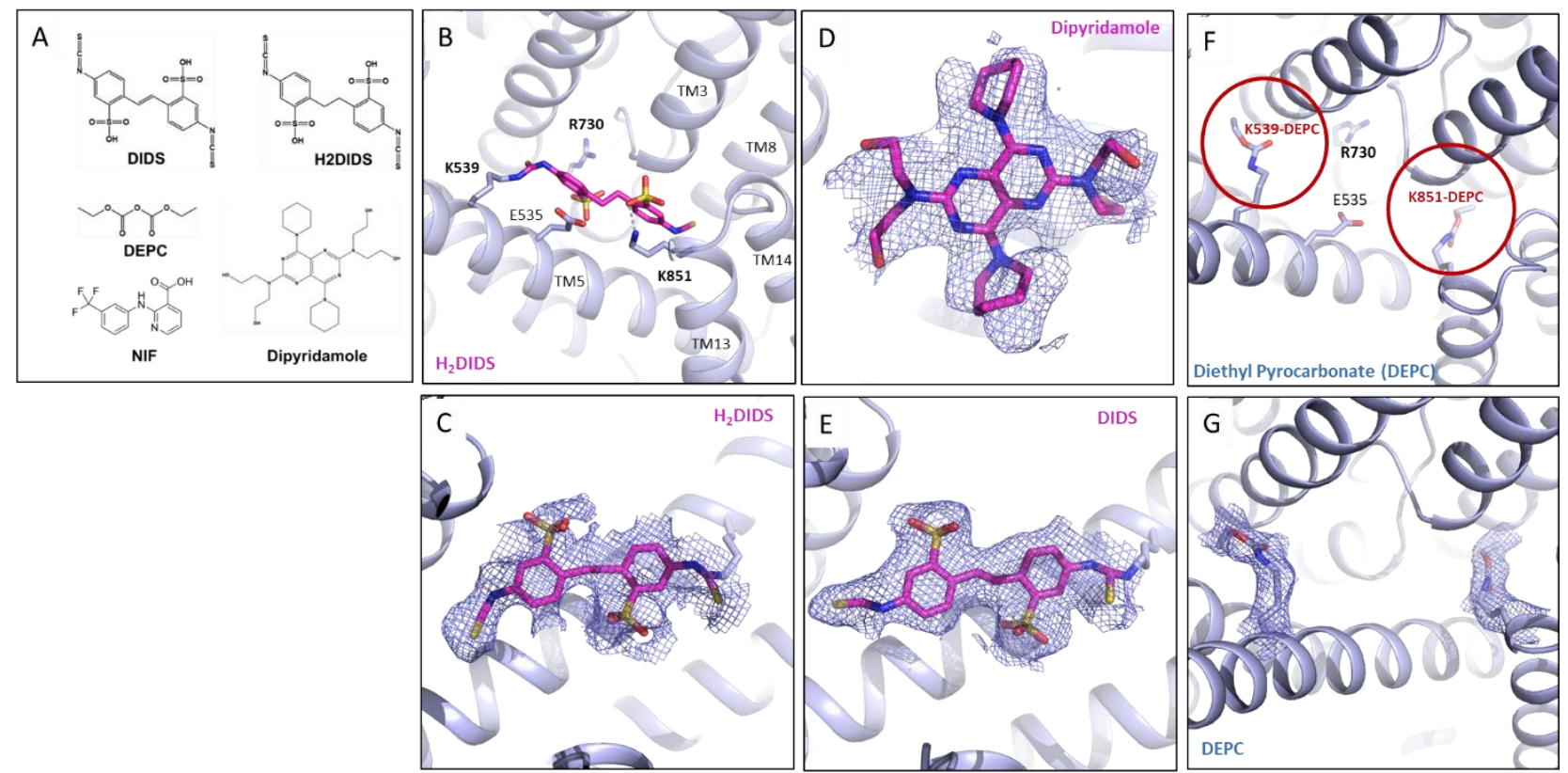

\section{Extended Data Fig. 3 | Structure and density of AE1-bound inhibitors. A, 2D} (magenta) location and cryo-EM density at a contour level of $3 \sigma$. D, Cryo-EM density of Dipyridamole (contour level of $4 \sigma$ ). E, Cryo-EM density of DIDS (contour level of $3.5 \sigma$ ). 

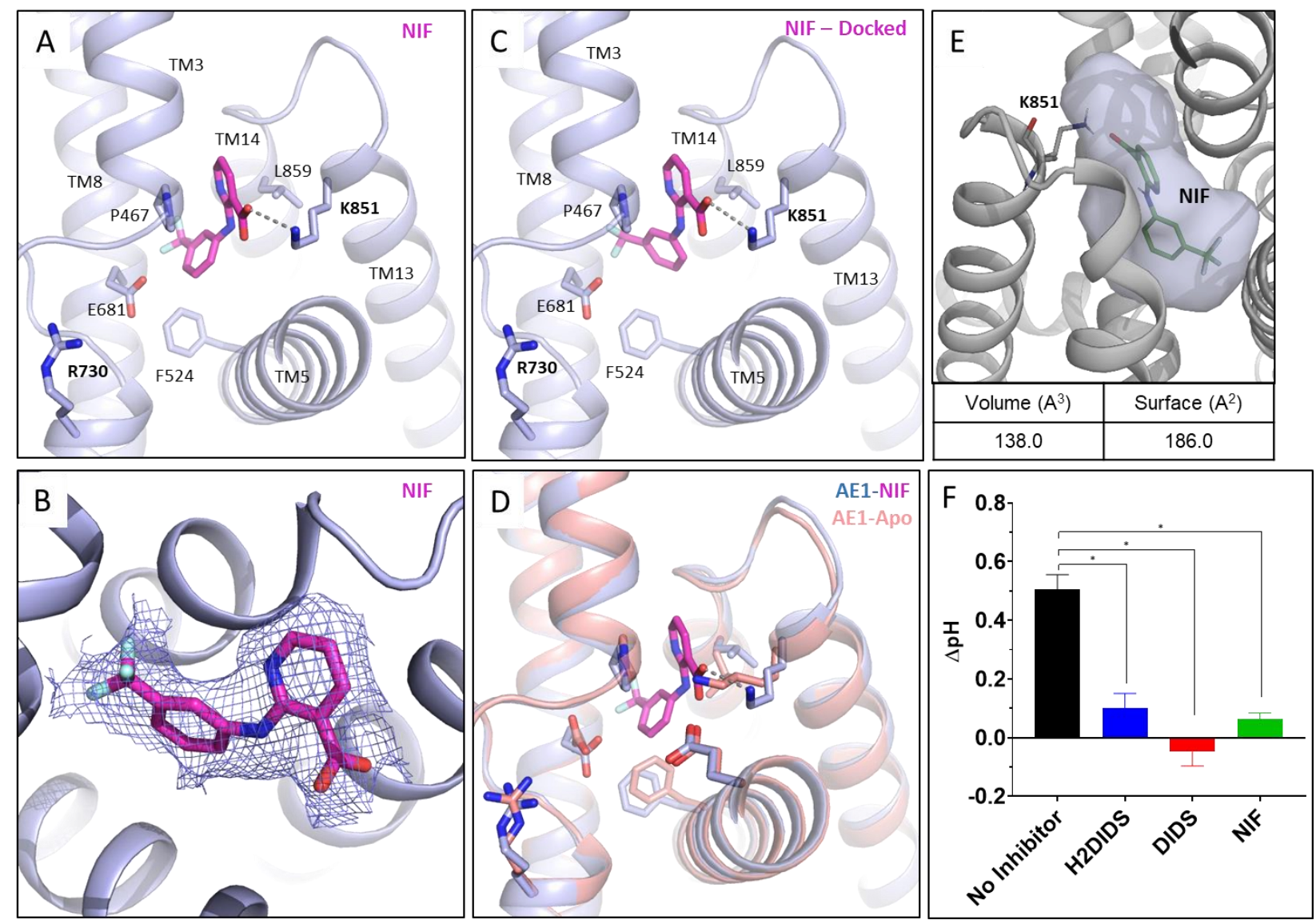

Extended Data Fig. 4 | NIF binding and experimental validation of AE1-inhibition

by $\mathrm{H}_{2}$ DIDS, DIDS, and NIF. A, B, NIF binding site and cryo-EM density shown at a contour level of $3.5 \sigma$. C, Validation of NIF binding by molecular docking, with the best scoring docking pose shown ( $\Delta \mathrm{G}$ binding score: -52.33$)$.

D, Overlay of Apo (salmon) and NIF-bound AE1 (light blue) reveals subtle conformational rearrangements required for NIF binding. E, Calculation of NIF binding pocket volume and surface in POVME. lonic interactions are shown as dotted lines. F, Determination of cellular bicarbonate uptake in the absence and presence of inhibitors (see methods for details). Dipyridamole could not be tested due to spectral overlap with the dye used to measure cellular $\mathrm{pH}$ changes. Data are mean \pm s.e.m. of three independent experiments $(n=3)$ performed in triplicates. One-way ANOVA was used to compare inhibited and uninhibited uptake $(p<0.0001)$. 
769 Extended Data Table 1 | Cryo-EM data collection, refinement and validation

770

statistics.

\begin{tabular}{|c|c|c|c|c|}
\hline & $\begin{array}{l}\text { AE1- } \\
\text { Apo } \\
(\text { EMDB-xxxx) } \\
(\text { PDB xxxx) }\end{array}$ & $\begin{array}{l}\text { AE1- } \\
\text { Bicarbonate } \\
(\text { EMDB-xxxx) } \\
(\text { PDB xxxx) } \\
\end{array}$ & $\begin{array}{l}\text { AE1- } \\
\text { DIDS } \\
(\text { EMDB-xxxx) } \\
(\text { PDB } x x x x) \\
\end{array}$ & $\begin{array}{l}\text { AE1- } \\
\mathrm{H}_{2} \text { DIDS } \\
(\text { EMDB-xxxx) } \\
(\text { PDB xxxx) } \\
\end{array}$ \\
\hline $\begin{array}{l}\text { Data collection and } \\
\text { processing }\end{array}$ & & & & \\
\hline $\begin{array}{l}\text { Magnification } \\
\text { Voltage }(\mathrm{kV}) \\
\text { Electron exposure }\left(\mathrm{e}-/ \AA^{2}\right) \\
\text { Defocus range }(\mu \mathrm{m}) \\
\text { Pixel size }(\AA) \\
\text { Symmetry imposed } \\
\text { Initial particle images (no.) } \\
\text { Final particle images (no.) } \\
\text { Map resolution }(\AA) \\
\quad \text { FSC threshold } \\
\text { Map sharpening B-factor }\left(\AA^{2}\right)\end{array}$ & $\begin{array}{l}64,000 \\
300 \\
51.85 \\
-0.5 \text { to }-1.8 \\
1.076 \\
\text { C2 } \\
4357888 \\
238474 \\
2.99 \\
0.143 \\
-150.0\end{array}$ & $\begin{array}{l}64,000 \\
300 \\
59.99 \\
-0.5 \text { to }-1.8 \\
1.076 \\
\text { C2 } \\
2660401 \\
173471 \\
3.37 \\
0.143 \\
-150.4\end{array}$ & $\begin{array}{l}81,000 \\
300 \\
52.09 \\
-0.5 \text { to }-1.8 \\
1.083 \\
\text { C2 } \\
5921308 \\
280284 \\
2.96 \\
0.143 \\
-138.7\end{array}$ & $\begin{array}{l}64,000 \\
300 \\
51.85 \\
-0.5 \text { to }-1.8 \\
1.076 \\
\text { C2 } \\
2460255 \\
267008 \\
2.98 \\
0.143 \\
-121.2\end{array}$ \\
\hline Refinement & & & & \\
\hline $\begin{array}{l}\text { Model composition } \\
\text { Non-hydrogen atoms } \\
\text { Protein residues } \\
\text { Ligands }\end{array}$ & $\begin{array}{l}8665 \\
1032 \\
14\end{array}$ & $\begin{array}{l}8584 \\
1034 \\
15\end{array}$ & $\begin{array}{l}8727 \\
1032 \\
16\end{array}$ & $\begin{array}{l}8728 \\
1032 \\
16\end{array}$ \\
\hline $\begin{array}{l}\text { R.m.s. deviations } \\
\text { Bond lengths }(\AA) \\
\text { Bond angles }\left(^{\circ}\right)\end{array}$ & $\begin{array}{l}0.086 \\
1.821\end{array}$ & $\begin{array}{l}0.014 \\
1.653\end{array}$ & $\begin{array}{l}0.05 \\
1.701\end{array}$ & $\begin{array}{l}0.013 \\
1.647\end{array}$ \\
\hline $\begin{array}{l}\text { Validation } \\
\text { Clashscore } \\
\text { Poor rotamers }(\%)\end{array}$ & $\begin{array}{l}3.49 \\
0.49\end{array}$ & $\begin{array}{l}4.92 \\
1\end{array}$ & $\begin{array}{l}1.12 \\
0.33\end{array}$ & $\begin{array}{l}4.45 \\
1.44\end{array}$ \\
\hline $\begin{array}{l}\text { Ramachandran plot } \\
\text { Favored }(\%) \\
\text { Allowed (\%) } \\
\text { Disallowed (\%) }\end{array}$ & $\begin{array}{l}94.04 \\
5.86 \\
0\end{array}$ & $\begin{array}{l}94.53 \\
5.47 \\
0\end{array}$ & $\begin{array}{l}96.09 \\
3.91 \\
0\end{array}$ & $\begin{array}{l}95.61 \\
4.39 \\
0\end{array}$ \\
\hline
\end{tabular}

771

772

773

774 


\begin{tabular}{|c|c|c|c|}
\hline & $\begin{array}{l}\text { AE1- } \\
\text { DEPC } \\
(\text { EMDB-xxxx) } \\
(\text { PDB xxxx) }\end{array}$ & $\begin{array}{l}\text { AE1- } \\
\text { Dipyridamole } \\
(\text { EMDB-xxxx) } \\
(\text { PDB xxxx) } \\
\end{array}$ & $\begin{array}{l}\text { AE1- } \\
\text { NIF } \\
\text { (EMDB-xxxx) } \\
\text { (PDB xxxx) }\end{array}$ \\
\hline $\begin{array}{l}\text { Data collection and } \\
\text { processing }\end{array}$ & & & \\
\hline $\begin{array}{l}\text { Magnification } \\
\text { Voltage }(\mathrm{kV}) \\
\text { Electron exposure }\left(\mathrm{e}-/ \AA^{2}\right) \\
\text { Defocus range }(\mu \mathrm{m}) \\
\text { Pixel size }(\AA) \\
\text { Symmetry imposed } \\
\text { Initial particle images (no.) } \\
\text { Final particle images (no.) } \\
\text { Map resolution }(\AA) \\
\text { FSC threshold } \\
\text { Map sharpening B-factor }\left(\AA^{2}\right)\end{array}$ & $\begin{array}{l}81,000 \\
300 \\
51.18 \\
-0.5 \text { to }-1.8 \\
1.083 \\
\text { C2 } \\
3156841 \\
191625 \\
3.07 \\
0.143 \\
-128.7\end{array}$ & $\begin{array}{l}64,000 \\
300 \\
51.69 \\
-0.5 \text { to }-1.8 \\
1.076 \\
\text { C2 } \\
2499027 \\
146097 \\
3.19 \\
0.143 \\
-130.1\end{array}$ & $\begin{array}{l}64,000 \\
300 \\
59.99 \\
-0.5 \text { to }-1.8 \\
1.076 \\
\text { C2 } \\
2977492 \\
79981 \\
3.18 \\
0.143 \\
-107.4\end{array}$ \\
\hline Refinement & & & \\
\hline $\begin{array}{l}\text { Model composition } \\
\text { Non-hydrogen atoms } \\
\text { Protein residues } \\
\text { Ligands }\end{array}$ & $\begin{array}{l}8674 \\
1028 \\
16\end{array}$ & $\begin{array}{l}8647 \\
1032 \\
16\end{array}$ & $\begin{array}{l}8578 \\
1032 \\
16\end{array}$ \\
\hline $\begin{array}{l}\text { R.m.s. deviations } \\
\text { Bond lengths }(\AA) \\
\text { Bond angles }\left(^{\circ}\right)\end{array}$ & $\begin{array}{l}0.027 \\
1.969\end{array}$ & $\begin{array}{l}0.014 \\
1.653\end{array}$ & $\begin{array}{l}0.013 \\
1.652\end{array}$ \\
\hline $\begin{array}{l}\text { Validation } \\
\text { Clashscore } \\
\text { Poor rotamers }(\%)\end{array}$ & $\begin{array}{l}3.91 \\
0.22\end{array}$ & $\begin{array}{l}4.19 \\
0\end{array}$ & $\begin{array}{l}6.88 \\
1.89\end{array}$ \\
\hline $\begin{array}{l}\text { Ramachandran plot } \\
\text { Favored (\%) } \\
\text { Allowed (\%) } \\
\text { Disallowed (\%) }\end{array}$ & $\begin{array}{l}95.75 \\
4.25 \\
0\end{array}$ & $\begin{array}{l}96.97 \\
2.83 \\
0.2\end{array}$ & $\begin{array}{l}96.00 \\
4.00 \\
0\end{array}$ \\
\hline
\end{tabular}



the AE1 anion binding site.

\begin{tabular}{|c|c|}
\hline Residue & Interaction Energy (kcal/mol) \\
\hline S465 & -0.19 \\
\hline I528 & -0.62 \\
\hline S529 & 0.12 \\
\hline I531 & -0.17 \\
\hline F532 & -0.25 \\
\hline E681 & 0.37 \\
\hline A726 & -0.46 \\
\hline T727 & -6.66 \\
\hline T728 & -2.55 \\
\hline V729 & -3.97 \\
\hline R730 & -11.33 \\
\hline S731 & -0.38 \\
\hline V732 & -0.15 \\
\hline T733 & -0.21 \\
\hline M795 & -0.13 \\
\hline
\end{tabular}


790 Extended Data Table 3 | Calculated pocket volume of bicarbonate and inhibitors

791 bound to AE1.

\begin{tabular}{|c|c|c|}
\hline Ligand & Volume $\left(\AA^{3}\right)$ & Surface $\left(\AA^{2}\right)$ \\
\hline Bicarbonate & 23 & 32 \\
\hline H2DIDS & 312 & 341 \\
\hline DIDS & 350 & 331 \\
\hline Dipyridamole & 447 & 373 \\
\hline Niflumic Acid & 138 & 186 \\
\hline
\end{tabular}

